\title{
INDUSTRIAL SEMISOLID CASTING PROCESS FOR SECONDARY ALUMINIUM ALLOYS FOR DECARBONISING LIGHTWEIGHT PARTS IN AUTOMOTIVE SECTOR
}

\author{
Fabrizio D’Errico ${ }^{1, *}$, Daniele Casari ${ }^{2}$, Mattia Alemani ${ }^{2}$, Guido Perricone ${ }^{2}$, Mauro Tosto ${ }^{2}$ \\ ${ }^{1}$ Politecnico di Milano, Department of Mechanical Engineering, 20156 Milan, Italy \\ ${ }^{2}$ Brembo SpA, 24040, Stezzano, Italy
}

\begin{abstract}
The life cycle holistic approach for the automotive sector highlighted how much important is working on decarbonisation of $\mathrm{Al}$ casting processes to produce vehicle components. Broadening the use of recycled aluminium alloys, instead of high energy intensive primary aluminium alloys, is key for environment preservation. While primary aluminium alloys are preferred by automotive original equipment manufacturers (OEMs) because impurities (mainly Fe) present in secondary aluminium alloys might cause fatigue properties decay, a new semisolid state process route has been developed at Brembo to drastically reduce the sensitivity of cast aluminium to $\mathrm{Fe}$ impurities. Based on these premises, during the CRAL European project in the framework of the LIFE Programme, an industrial prototype machinery - a vertical high pressure die casting machine - has been designed and constructed to inject secondary aluminium in the semisolid state. A number of A357 Fe-enriched brake callipers manufactured via the new process route have been fully investigated by fatigue tests, SEM and OM analyses in order to validate the new eco-sustainable product compared to conventional ones manufactured with primary aluminium by gravity casting.
\end{abstract}

\section{Introduction}

It is common knowledge today that lightweight vehicles are fundamental for decreasing traffic-related pollutant emissions by conventional internal combustion engine powered vehicles. The EU legislation is continuously evolving for more restrictive emission targets for new vehicles. Different studies show potential weight reduction scenarios going from $15 \%$ to $35 \%$ weight reduction $[1,2]$, estimating a potential greenhouse gas emissions reduction in the range 9-18 Gtons CO2eq between 2010 and 2050 [5]. Reducing unsprung weight onboard vehicles is the key to improving handling, reducing inertia adverse effect, improving powertrain efficiency. The lower the unsprung weight, the less work the shocks and springs have to do to keep the tires in contact with the road over bumpy surfaces. Lot of problems, if not all of them, is caused in fact by inertia. Heavier unsprung mass (such as brake assemblies, tires, hubs, knuckles) means higher inertia; the higher the inertial dynamic forces, the more is the energy consumed by suspension spring and dampers for accommodating shocks to keep tyres on the ground. Similarly, if unsprung components have a high mass, they are harder to accelerate/decelerate and thus it is more difficult for the suspension to maintain a consistent tyre load.

Reducing mass in these locations improves the vehicle handling and performance, and provides fuel efficiency [3]; it is estimated that cutting one kilogram of unsprung weight is comparable, due to vehicle dynamics, to reducing the sprung mass weight by $3-6 \mathrm{~kg}$ (depending on inertial energy absorbed by dampers, i.e. on road irregularities). The effect of less unsprung weight means that the vehicle's suspension can control the wheel and, in turn, the tyres' contact patch more effectively, resulting in better traction and vehicle control. Reducing the weight of the braking system has been a key area of focus for automobile manufacturers.

The manufacturers are reducing the unsprung weight of today's modern vehicles everywhere they can and these lighter assemblies give the driver more effective cornering, handling, braking, and improved road feel.

A key role in this challenge is played by lightweight materials for structural and massive components such as aluminium alloys. Old cast-iron brake calipers have been replaced by ones made of aluminium.

Nevertheless, aluminium alloys currently used in conventional casting processes for manufacturing lightweight unsprung subsystem components such as knuckles and brake system assemblies suffer from environmental drawbacks over the whole cradle-to-grave cycle. Specifically, high-purity primary aluminium is required to produce the final casting alloys and the extraction process of primary aluminium is an energy consuming process with a high environmental impact [4$6]$.

If on one hand, weigthsaving by putting onboard lighter aluminium components instead of heavier ones can lower the fuel consumption and pollutant emissions per

* Corresponding author: fabrizio.derrico@polimi.it 
kilometre for all internal combustion engine vehicles, on the other hand such benefit could not be fully achieved if a negative net balance for the total $\mathrm{CO} 2$ emitted over the manufacturing phases occurred.. More specifically, the use of secondary aluminium alloys derived from recycling patterns instead of primary aluminium alloys would allow a 95\% net energy saving and a $80 \%$ reduction in total greenhouse gases emissions, avoiding any type of chemicals $[7,8]$. Therefore, it is clear that a wider use of secondary alloys in casting components for the automotive sector is an environmentally conscious choice for a significant cleaning-up of the vehicles' manufacturing stage. Unfortunately, original equipment manufacturer (hereinafter OEMs for brevity) use mostly primary Al-based alloys. However, this choice is due to the fact that the use of recycled aluminium could introduce some metal impurities such as iron and manganese, residual of recycling patterns. Such impurities are detrimental for the toughness of final cast components because they cause the precipitation, during the solidification phase, of hazardous needle-like or blocky phases [9-12]. Conventional casting technologies such as gravity casting or low and high pressure die casting produce coarse intermetallic $\beta$-Al5FeSi platelets, resulting in poorer mechanical properties.

\subsection{Effect of shear on Fe intermetallic formation}

The Fe tolerance of Al-Si based alloys is closely related to the morphology of the primary Fe-containing intermetallic compounds, and the detrimental effect of Fe can be reduced or even eliminated by the removal of the large needle-shaped primary $\beta$-Al5FeSi compounds. Despite being just a few, research studies exist in which it is shown that shearing improves the Fe tolerance of Al-Si-based casting alloys [13]. Fan et al. claim that Rheo die cast processes (hereinafter RDC for brevity) can increases the tolerance to $\mathrm{Fe}$ contents [14]. RDC samples can tolerate a Fe concentration up to 1.5 wt.\% without any severe damages to the mechanical properties. This is because intensive shearing changes the morphology of iron-containing compounds from long needles or plates to much smaller particles.

Fang et al [13] investigated the effects of intensive forced-melt convection, generated by RDC processing, on the mechanical properties of Fe-containing $\mathrm{Al}-\mathrm{Si}$ alloys, noticing some interesting aspects. Their activity on the LM25 commercial alloy, to which different amounts of iron were added, showed that RDC is effective in modifying the morphology of the primary Fe-bearing phases. Such processing route appears to be effective in spheroidising both the $\alpha-A l$ and the monoclinic $\beta-\mathrm{A} 15 \mathrm{FeSi}$ phases. An intensive melt shearing in the semisolid state is effective in turning the long needle morphology into less detrimental short bars or discs with a substantial reduction in the aspect ratio. An explanation for the mentioned effect is also given [14]. They believe that the formation of a needle-shaped morphology is owed to a significantly different solidliquid (S-L) interfacial energy and to the associated disparity in growth rates along different crystallographic directions. Consequently, the modification of aspect ratios for the $\beta$-Al5FeSi phase suggests that melt shearing could reduce the differences in solid-liquid interfacial energies and in turn slow down preferential growth in the direction of the longitudinal needle axis.

\subsection{The CRAL project}

The 3 and $1 / 2$ years European Commission co-financed LIFE CRAL project, coordinated by Brembo Braking System Spa, ended in December 2019. It was aimed at designing and validating a novel industrial semisolid process plant that would have used low-purity (i.e. Feenriched) Al alloys to manufacture lightweight highperformance brake callipers, reducing the total cradle-togate carbon footprint compared to primary aluminium brake callipers manufactured by the conventional gravity casting process.

Based on previous literature $[13,14]$, the improvement of the semisolid casting technology was considered as a key aspect to reach such objectives.

The semisolid process adopted for the industrial pilot plant is based on the rheocasting process route, which consists in stirring a metallic alloy in a temperature range where solid and liquid particles coexist, i.e. during the first stage of solidification.

The stirring and controlled cooling actions change the initial dendritic solid particles into globular ones, leading to a more homogeneous microstructure.

The pilot semi-solid method (hereinafter SSM for brevity) line is shown in Fig.1. It is a completely automated die casting area equipped with a stationary holding furnace, two anthropomorphic robotised arms and a $10.000 \mathrm{kN}$ 4-columns vertical die-casting press. The 3D render of the press is shown in Fig.2. The press is equipped with a rotating plate having three containers, each for a specific process stage. When the container is in the first position, namely out of the press moving plate, the container works as a pouring chamber: the pouring robot picks up the aluminium alloy from the holding furnace and pours it into the container. Then, the stirring device enters the container and starts mixing the molten metal while the cooling system allows to control the temperature into the solidification interval.

Subsequently, the container rotates under the moving plate, mechanically coupling with the injection piston and preparing for the injection of the semisolid metal.

The injection pressure nominally available for the CRAL press machinery is $3.000 \mathrm{kN}$, capable of injecting the liquid metal with a 1.800 bar metal pressure. Fig. 3 displays the rheo-cast component manufactured via the CRAL pilot SSM line, i.e. a specifically developed monoblock brake calliper.

Microstructural investigations as well as bench and road tests were then performed in order to assess the performance of the component. The interactions of the brake calliper with the wheel corner and the suspensions were taken into account during these tests as well as the vehicle dynamics and vibration modes that could lead to unwanted noise generation (Fig.4). 


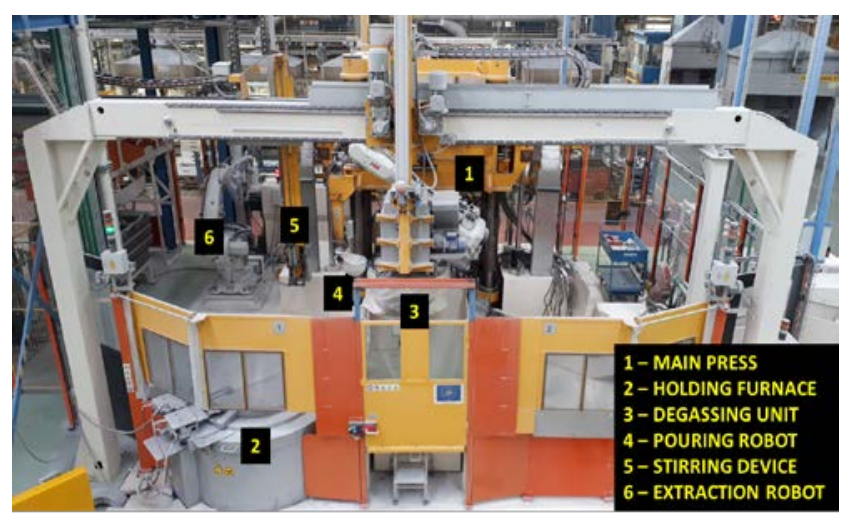

Figure 1. The CRAL semisolid rheo-casting plant.

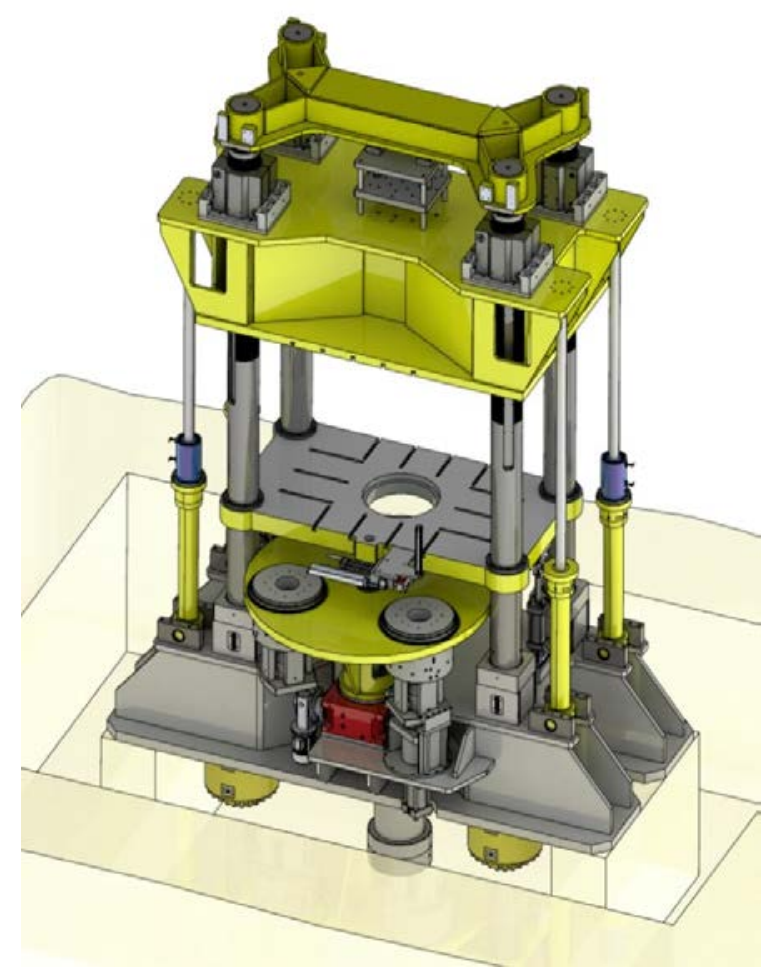

Figure 2. The CRAL vertical rheo-casting press machinery.

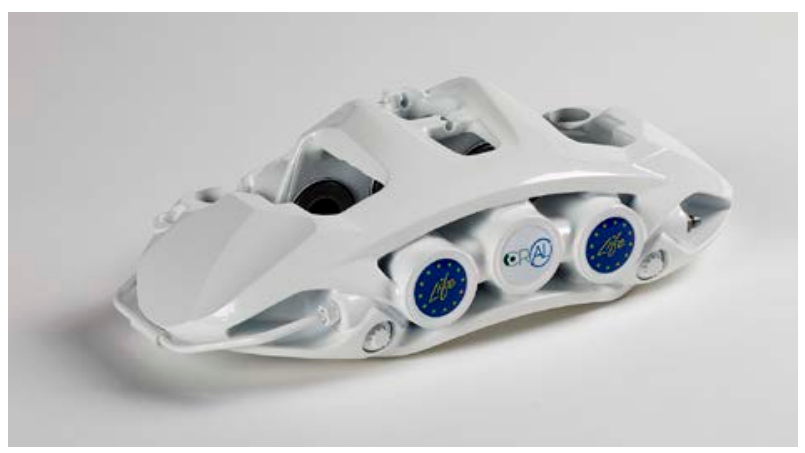

Figure 3. Demonstrator brake calliper made of secondary aa356 alloy by semisolid high pressure die casting process.

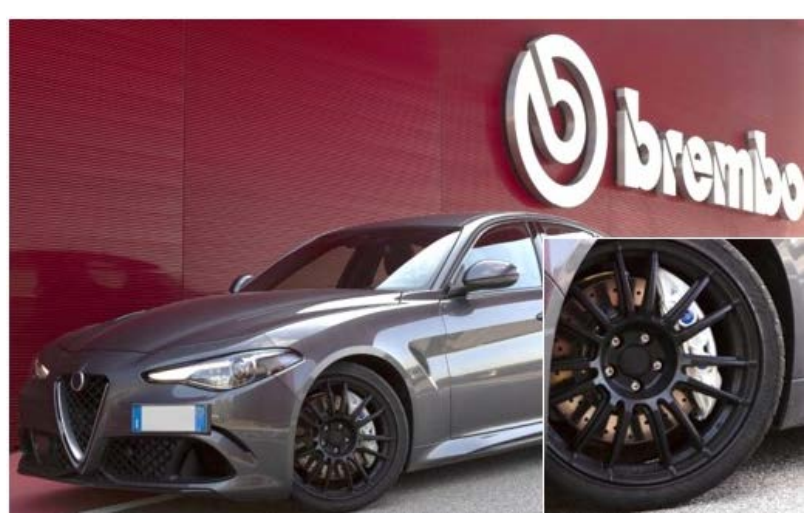

Figure 4. The CRAL secondary Al calliper mounted on wheel hub for road tests.

\section{Experimental part}

The material used for the test campaign is an A357 Brembo-type alloy with a $0.80-0.95$ wt. $\%$ Fe concentration. In order to fulfil OEMs specifications for new products, the Brembo team focused on a number of process parameters considered as crucial for improving the metallurgical quality of the parts. It was found by several tests that the main parameters to control during the CRAL process can be divided into two groups, the out-of-press and in-press parameters, such as:

- Out-of-press parameters: pouring temperature and stirring time of the semisolid slurry. Several trial tests were carried out to evaluate heat losses and temperature decrease occurring when the liquid metal is transferred from the holding furnace to the thermoregulated containers. Three different starting temperatures were investigated. Experiments aimed at clarifying the evolution of the solid fraction as a function of stirring time were performed..

- In-press parameters: temperature of the mould, plunger speed and intensification pressure.: Casting trials were performed in order to determine the optimal injection parameters After the process parameters were fine-tuned, a small batch of 100 brake callipers was produced.

During casting trials, the defects observed in the cast parts were summarised in a preliminary list in order to have an inventory of metallurgical defects that could correlate with process parameters. 
Table 1. Major metallurgical defects observed in preliminary casting trials.

\begin{tabular}{|c|c|c|}
\hline Issue & $\begin{array}{l}\text { Parameters } \\
\text { influencing } \\
\text { defects }\end{array}$ & Undertaken action \\
\hline \multirow{2}{*}{$\begin{array}{l}\text { Shrinkage } \\
\text { porosities }\end{array}$} & geometry & Modified geometry \\
\hline & $\begin{array}{l}\text { solidification } \\
\text { kinetics }\end{array}$ & Optimisation of injection curves \\
\hline \multirow{3}{*}{$\begin{array}{l}\text { Oxides and } \\
\text { Blisters }\end{array}$} & $\begin{array}{l}\text { Metallurgical } \\
\text { cleanliness }\end{array}$ & Modified lubricant \\
\hline & Air entrapment & \multirow[b]{2}{*}{$\begin{array}{l}\text { Study on specific heat treatments } \\
\text { and/or post heat treatments }\end{array}$} \\
\hline & $\begin{array}{l}\text { Lubrication } \\
\text { system/lubricant } \\
\text { type }\end{array}$ & \\
\hline
\end{tabular}

A number of samples were extracted from specific regions of the CRAL secondary Al callipers (Figure 5) and subsequently machined in conformity with internal drawings to obtain tensile specimens.

The outcome of tensile tests is reported in Table 2. Results from CRAL primary $\mathrm{Al}$ specimens and samples extracted from gravity cast callipers are also included. It can be observed that the CRAL process allows for: (i) enhanced mechanical properties (CRAL primary Al vs. gravity casting); (ii) adequate performances even if secondary $\mathrm{Al}$ alloys are employed.

Figure 6 shows the representative microstructures of primary $\mathrm{Al}$ gravity cast and secondary $\mathrm{Al}$ semi-solid samples, respectively. Primary $\alpha-\mathrm{Al}$ and $\mathrm{Al}-\mathrm{Si}$ eutectic phases can be observed.

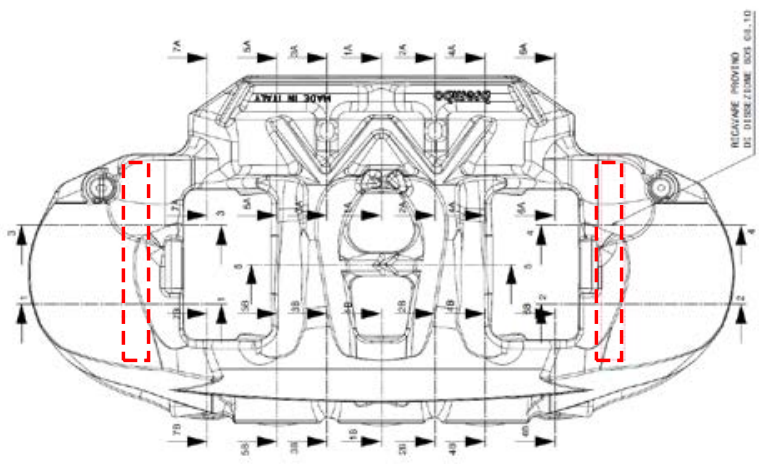

Figure 5. Technical drawing of the CRAL secondary Al calliper; the tensile specimens are extracted from the regions marked with dashed red lines, while the black arrows indicate the sections undergoing standard metallographic investigations in Brembo aluminium foundry Quality Lab.

Table 2. Comparison between the tensile properties of specimens extracted, respectively, from gravity cast, CRAL primary $\mathrm{Al}$ and CRAL secondary $\mathrm{Al}$ callipers.

\begin{tabular}{|c|c|c|c|}
\hline Alloy & $\begin{array}{c}\mathbf{R p}_{\mathbf{.} . \mathbf{2}} \\
{[\mathbf{M P a}]}\end{array}$ & $\mathbf{R m}[\mathbf{M P a}]$ & $\mathbf{A} \%$ \\
\hline Gravity casting & $321 \pm 22$ & $285 \pm 17$ & $2.95 \pm 1.68$ \\
\hline CRAL primary Al & $\geq 320$ & $\geq 280$ & $\geq 3$ \\
\hline CRAL secondary Al & $\geq 290$ & $\geq 250$ & $\geq 1$ \\
\hline
\end{tabular}

All the specimens exhibit a very homogeneous microstructure, globular and rosette-type finely distributed grains. Fe-bearing intermetallic platelets can also be noted on the fracture surface of broken samples (Fig. 7). These particles are well known in literature to be highly detrimental to the mechanical properties of aluminium alloys.

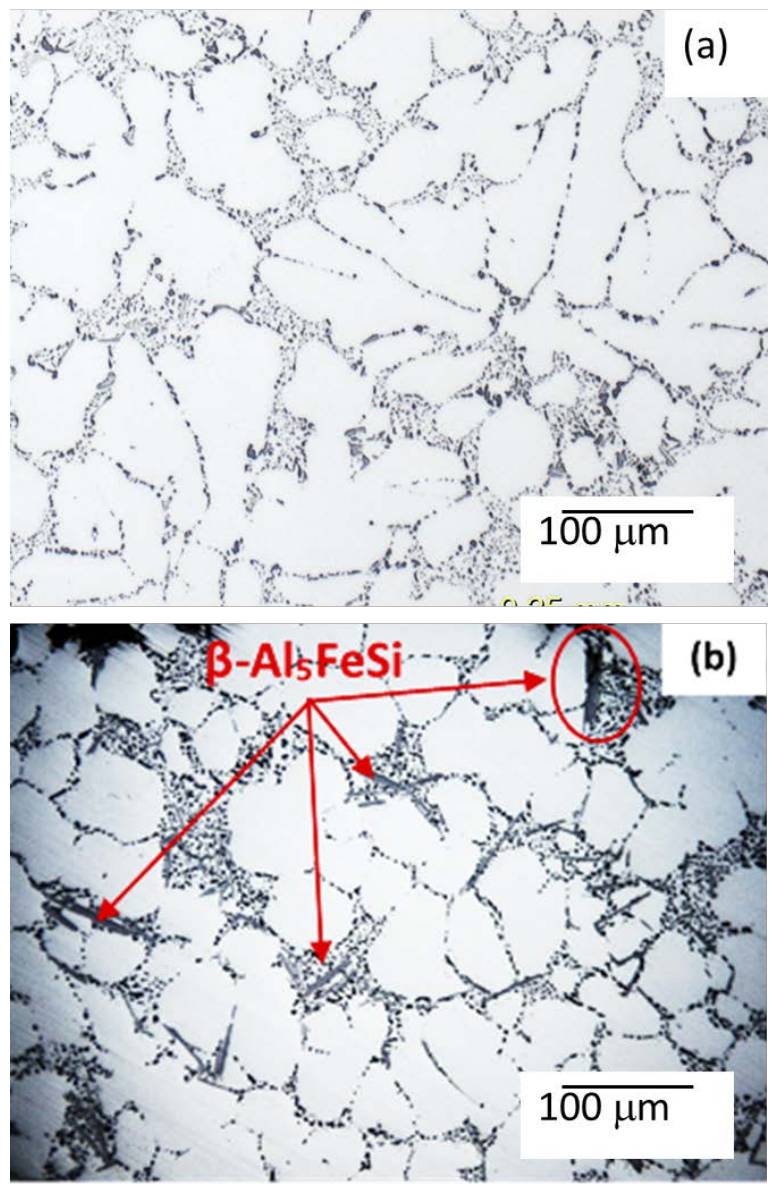

Figure 6. Microstructures at low and high magnification: a) gravity casting, conventional process; b) CRAL-secondary Al casting. Arrows identify the $\beta$-Al5FeSi phase. Such particles have a crucial role in the mechanical behaviour of the alloy, prompting fracture initiation and subsequent propagation even at low applied loads (cracked particle marked with the red oval).

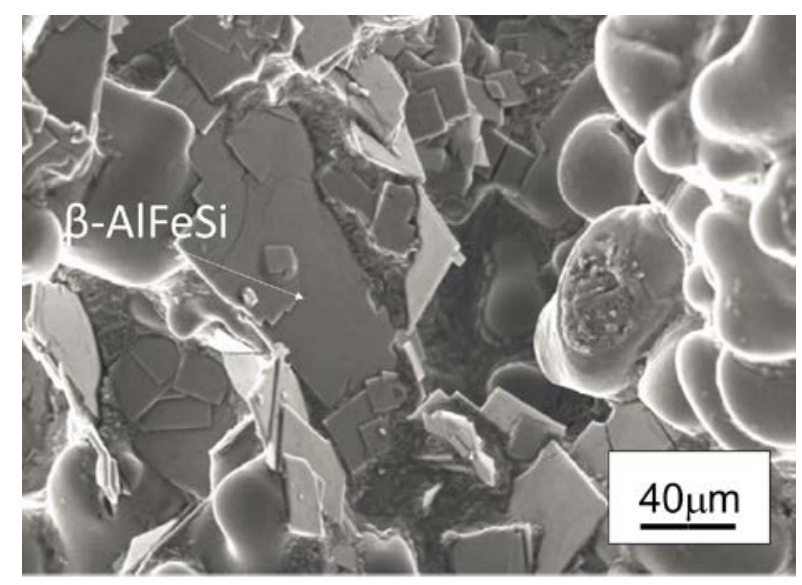

Figure 7. SEM image of the fracture surface of a tensile specimen revealing the presence of Fe-bearing intermetallic phases. 
Eventually, the Design and Verification Plan (DVP) ensured that the prototype callipers responded to usual operating conditions, especially in case of emergency braking. Table 3 shows the different kinds of bench-tests carried out on the CRAL secondary Al callipers, all successfully passed (Fig.8). The DVP also included road tests: about $1000 \mathrm{~km}$ were covered on Italian roads in order to validate the brake calliper for the use on vehicles.

Table 3. CRAL secondary Al calliper Design and Verification Plan.

\begin{tabular}{|c|c|}
\hline \multicolumn{2}{|c|}{ Basic Characteristics } \\
\hline BTS 61.01 & Air Pressure Seal Test \\
\hline BTS 61.02 & Hydraulic Pressure Tightness \\
\hline BTS 61.03 & Vacuum Tightness \\
\hline BTS 61.21 & Bleedability \\
\hline \multicolumn{2}{|c|}{ Functionality } \\
\hline BTS 61.06 & Fluid Displacement \\
\hline BTS 61.07 & Luft - Roll-Back \\
\hline BTS 61.20 & Dragging Torque \\
\hline BTS 61.07 & Knock-back \\
\hline BTS 61.24 & Squeak Test \\
\hline BTS 61.07 & Piston sliding pressure \\
\hline BTS 61.07 & Pistons return load \\
\hline BTS 61.04 & Fluid Pressure Stiffness \\
\hline \multicolumn{2}{|l|}{ Strength } \\
\hline BTS 61.05 & Burst pressure \\
\hline BTS 61.08 & Torque Strength \\
\hline BTS 61.22 & Strength of coupling and screw-bleeder \\
\hline \multicolumn{2}{|l|}{ Durability } \\
\hline BTS 61.19 & Vibration Test \\
\hline BTS 61.11 & Pulsating Pressure Endurance \\
\hline BTS 61.11 & Pulsating pressure endurance - Temp. \\
\hline BTS 61.12 & $\begin{array}{l}\text { Pressure and Torque Durability - Passenger } \\
\text { Car }\end{array}$ \\
\hline
\end{tabular}

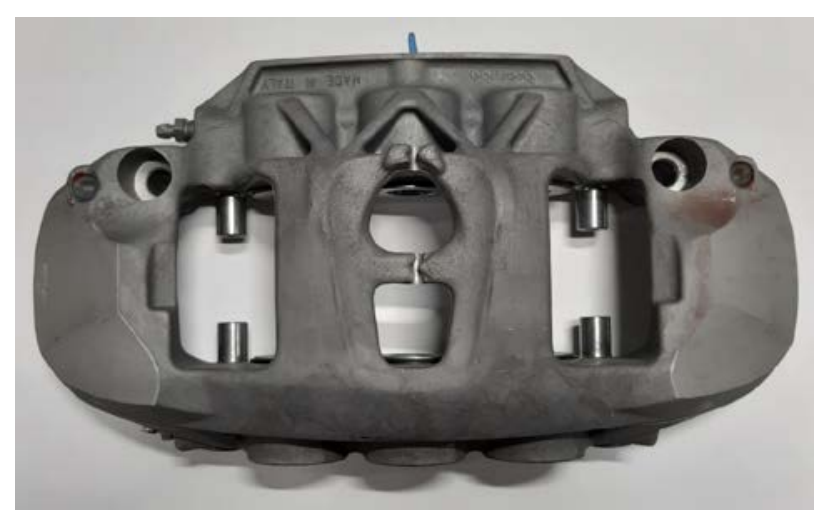

Figure 8. CRAL secondary $\mathrm{Al}$ calliper after pressure and torque durability test.

\section{Discussion}

In the recent years, many processes were investigated for modifying the harmful Fe-bearing intermetallic compounds by rapid solidification, addition of neutralising elements and melt superheating. Nevertheless, low efficiency, complicated processes or high costs accompany the above approaches. Few studies can be found in which the role of slurry stirring on the size and distribution of iron-based intermetallic compounds is investigated. The use of neutralising elements is by far the most common response to iron in the casting industry, but not the proper one. The Fe tolerance of $\mathrm{Al}-\mathrm{Si}$ alloys is closely related to the morphology of the primary Fe-containing intermetallic compounds. It has been demonstrated that the detrimental effect of $\mathrm{Fe}$ can be reduced or even eliminated by modifying the large needle-shaped primary $\beta$-Al5FeSi compounds via shearing [13].

Furthermore, the globular structure achieved by means of the CRAL process can counterbalance the harmful effect of Fe-containing platelets, allowing for mechanical properties in compliance with Brembo and OEMs standards. Such platelets were observed to be much smaller than those usually found in gravity cast components $(\sim 50 \mu \mathrm{m}$ vs. $200 \mu \mathrm{m})$, hence highlighting the advantage of semisolid stirring of the metal. Intensive melt shearing in the semisolid state is considered very effective in turning the long needle morphology into less detrimental short bars or discs with a substantial reduction in the aspect ratio. Further positive results over the intensive shearing is obtained by combined effect of shearing action and the intensive forced convection and the much more homogenous temperature in the mass during steering that help stabilisation of the $\alpha$-AlFeMnSi intermetallic compounds with respect to the $\beta$-AlFeSi.

In order to assess the efficiency of RDC processing in hindering the detrimental effect of $\mathrm{Fe}$ on mechanical properties of cast $\mathrm{Al}$ alloys, the concept of Fe tolerance was used in this manuscript; since the elongation of die cast components is the most significant property, being severely affected by impurities such as iron, this parameter was linked to the concept of Fe tolerance.

\section{Conclusions}

The high-pressure rheo-casting industrial process developed in the CRAL project appears to be consistent with the metallurgy phenomena indicating that the higher is the refinement of the primary globular cells, the lower is the growth rate of the intermetallic phases forming in the eutectic spaces (such as Fe-containing intermetallics in secondary $\mathrm{Al}-\mathrm{Si}$ alloys). Also, the stirring produces a more homogeneous microstructure, which can counteract the detrimental effects of $\mathrm{Fe}$ bearing intermetallic compounds, leading to reasonably good mechanical properties as well as performances in line with internal and OEMs standards. 
The Brembo team equipped a real vehicle with recycled aluminium brake callipers. The DVP results were very promising, since a reasonably good mechanical response was obtained for recycled pressure-die casting aluminium brake callipers. This represents a breakthrough for the whole automotive industry, which will be also extended to further Brembo core products.

\section{Acknowledgements}

This work makes use of results produced by the CRAL project (http://www.cralproject.eu/en), co-funded by the European Commission within the 2015 LIFE+ Programme. Authors thank support provided by European Commission for supporting project dissemination activities. This work reflects only the authors' views. The Community is not liable for any use that may be made of the information contained therein.

\section{References}

1. McKinsey \& Company, Lightweight, Heavy Impact (2012).

2. P. Martino, 4th Int. Conf. Advanced Materials and Tech. for Transport, Turin (2014)

3. A.K. Sachdev, R.K. Mishra, A. Mahato, A. Alpas, ICAA13, Pittsburgh (2012)

4. D.R. Gunasegaram, A. Tharumarajah, Met. and Mat. Trans. B 40 (4) (2009)

5. R.B.H. Tan,, H.H. Khoo, J. of Cleaner Prod., 13 (6) (2005)

6. B.J. Welch, M.M. Hyland, B.J. James, JOM, 53 (2) (2001)

7. T.E. Norgate, W.J. Rankin, Int. Symposium on Greenhouse Gases in the Metallurgical Industries: Policies, Abatement and Treatment (2001)

8. R.B.H. Tan, H.H. Khoo, J. of Cleaner Prod. 13 (6) (2005)

9. S.G. Irilzap and N. Saklakoglu, Eng. Sc. and Tech. 17 (2014)

10. Q.G Wang, D. Apelian, D.A Lados, J. Light Metals, 1 (1) (2001)

11. A. Fabrizi, G.Timelli, S. Ferraro, F. Bonollo, Int. J. of Materials Research, 106 (7) (2015)

12. L.A. Narayanan, F.H. Samuel, J.E. Gruzleski, Met. and Mat. Trans. A, 25 (8) (1994), 1761-1773

13. X. Fang, G. Shao, Y. Liu and Z. Fan, Mat. Sc. and Eng. A (2007)

14. Z. Fan, X. Fang and S. Ji, Mat. Sc. and Eng. A 412 (2005) 